\title{
105. Pseudo-compactness and Strictly Continuous Convergence
}

By Kiyoshi IséKI

(Comm. by K. KunUGI, M.J.A., Oct. 12, 1957)

In my Note [3], the present author gave a characterisation of countably compactness of weakly separable completely regular spaces by using the notion of continuous convergence. In this paper, we shall give necessary and sufficient conditions for pseudo-compactness of completely regular spaces.

First, we shall define two types of the convergences of functions on a topological spaces. One of them (see Definition 2) is due to C. Kuratowski [4]. Next, we note a trivial relation between these two notions for the completeness, and we shall treat some related topics. Finally, we shall state the main Theorems 2 and 3.

Definition 1. A sequence $\left\{f_{n}(x)\right\}$ on a topological space $S$ is said to be continuous convergent to $f(x)$, if $x_{n} \rightarrow x$ implies $f_{n}\left(x_{n}\right) \rightarrow f(x)$.

Definition 2. A sequence $\left\{f_{. n}(x)\right\}$ on a topological space $S$ is said to be strictly continuous convergent to $f(x)$, if the convergence of $\left\{f\left(x_{n}\right)\right\}$ implies the convergence of $\left\{f_{n}\left(x_{n}\right)\right\}$ and $\lim f_{n}\left(x_{n}\right)=\lim f\left(x_{n}\right)$.

Let $f_{n}(x)$ and $f(x)$ be continuous functions and suppose that $f_{n}(x)$ is strictly continuous convergent to $f(x)$. If $x_{n} \rightarrow x$ then $f\left(x_{n}\right) \rightarrow f(x)$ by the continuity of $f(x)$. Hence, since $f_{n}$ is strictly continuous convergent, $\lim f_{n}\left(x_{n}\right)=\lim f\left(x_{n}\right)=f(x)$. Therefore $f_{n}(x)$ converges to $f(x)$ continuously.

Conversely, let $S$ be a sequentially compact, and suppose that $f_{n}(x)$ converges continuously to $f(x)$, then we shall show that $f_{n}(x)$ is strictly continuous convergent to $f(x)$.

To show it, we shall suppose that $f_{n}$ is not strictly continuous convergent to $f$, there is a sequence $\left\{x_{n}\right\}$ such that $f\left(x_{n}\right)$ is convergent to a number $\alpha$ and $f_{n}\left(x_{n}\right)$ is not convergent to $\alpha$. Therefore we can find a subsequence $f_{n_{i}}\left(x_{n_{i}}\right)$ such that every subsequence of it does not converge to $\alpha$. Since $S$ is sequentially compact, $\left\{x_{n_{i}}\right\}$ contains a convergent sequence $\left\{x_{n_{i j}}\right\}$. Let $x$ be the limit point of $\left\{x_{n_{i j}}\right\}$. From the hypothesis, $\lim f_{n_{i_{j}}}\left(x_{n_{i_{j}}}\right)=f(x)$. By the continuity, we have $f\left(x_{n_{i_{j}}}\right) \rightarrow f(x)$ and $\lim f\left(x_{n_{i_{j}}}\right)=\lim f\left(x_{n}\right)=\alpha$. Hence we have $f(x)=\alpha$. This shows that $f_{n_{i_{j}}}\left(x_{n_{i_{j}}}\right) \rightarrow \alpha$, which is contradiction.

Next, we shall consider a completely regular space $S$, and suppose that every sequence of continuous functions which is convergent continuously is strictly continuous convergent. Then we shall show that $S$ is countably compact. Suppose that $S$ is not countably compact, then there is a countable set $\left\{a_{n}\right\}$ without cluster point. Therefore, for 
$\left\{a_{n}\right\}$, we can find a sequence of neighbourhood $U_{n}$ of $a_{n}$, such that $U_{m} \frown U_{n}=\phi$ for $m \neq n$. By the complete regularity of $S$, there are continuous functions $f_{n}(x)$ on $S$ such that

$$
f_{n}(x)= \begin{cases}0 & x \in U_{n} \\ 1 & x=a_{n}\end{cases}
$$

and $0 \leq f_{n}(x) \leq 1$ on $S$. By the definition of $\left\{f_{n}(x)\right\}, f_{n}(x)$ is convergent continuously to $f(x) \equiv 0$. We have $0=\lim f\left(a_{n}\right)=\lim f_{n}\left(a_{n}\right)$. On the other hand, $f_{n}\left(a_{n}\right)=1$ for each $n$, but this contradicts. Therefore we have the following

Theorem 1. For a weakly separable completely regular space S, the following conditions are equivalent:

1) $S$ is sequentially compact.

2) $S$ is countably compact.

3) If every sequence of continuous functions on $S$ is continuously convergent to a continuous function, then it is strictly continuous convergence to the continuous function.

We shall consider the relation between uniform convergence and strictly continuous convergence. First we shall suppose that a sequence $\left\{f_{n}(x)\right\}$ of continuous functions on a pseudo-compact space $S$ is strictly continuous convergence to a continuous function $f(x)$. We shall show that the convergence is uniformly. To do so, we shall suppose that the convergence is not uniformly. Then there are a positive $\varepsilon$, a sequence of indices $n_{1}<n_{2}<\cdots$ and a sequence of points $\left\{x_{i}\right\}$ in $S$ such that

$$
\left|f_{n_{i}}\left(x_{i}\right)-f\left(x_{i}\right)\right|>\varepsilon \quad \text { for every } i .
$$

Since $S$ is pseudo-compact and $f(x)$ is continuous, $\left\{f\left(x_{i}\right)\right\}$ is a bounded sequence. $\left\{f\left(x_{i}\right)\right\}$ contains a convergent subsequence $\left\{f\left(x_{i_{k}}\right)\right\}$. Let $\alpha$ be the limit of $\left\{f\left(x_{i_{k}}\right)\right\}$. By the hypothesis, $f_{n_{i_{k}}}\left(x_{i_{k}}\right) \rightarrow \alpha$. For some $i_{k}$, we have

and this is impossible.

$$
\left|f_{n_{i_{k}}}\left(x_{i_{k}}\right)-f\left(x_{i_{k}}\right)\right|<\varepsilon
$$

Conversely, if $f_{n}(x) \rightarrow f(x)$ for continuous functions is uniformly convergent on a topological space, then its convergence is strictly continuous. As the proof is very similar with the first part of Theorem 1, we shall omit it (for the detail, see C. Kuratowski [4, p. 109]). Let $S$ be a completely regular space, and suppose that every sequence $\left\{f_{n}(x)\right\}$ of continuous functions on $S$ which converges strictly continuous to a continuous function $f(x)$ is uniformly convergent to $f(x)$ on $S$. Then we shall prove that $S$ is pseudo-compact. To prove it, we shall suppose that there is an unbounded continuous function $f(x)$ and we can suppose that $f(x)$ is non-negative. We shall define continuous functions $f_{n}(x)$ as follows: 


$$
f_{n}(x)=\operatorname{Min}(f(x), n) .
$$

It is clear that $f_{n}(x)$ is not uniformly convergent to $f(x)$. On the other hand, the sequence $\left\{f_{n}(x)\right\}$ is strictly continuous convergent to $f(x)$ on $S$. To show it, suppose that $f\left(x_{n}\right) \rightarrow \alpha(n \rightarrow \infty)$, then, for some fixed integer $N$ such that $\alpha<N$, there is an index $K$ that $K \leq m$ implies $f\left(x_{m}\right)<N$ therefore, if $K \leq m$, then $f_{m}\left(x_{m}\right)=f\left(x_{m}\right)$. Hence $\lim f_{m}\left(x_{n}\right)=\lim f\left(x_{m}\right)$. This shows that $S$ is pseudo-compact. Therefore, we have the following characterisation of pseudo-compact spaces.

Theorem 2. For a completely regular space S, the following two propositions are equivalent:

1) $S$ is pseudo-compact.

2) If every sequence of continuous functions on $S$ converges strictly continuous to a continuous function, its convergence is uniformly on $S$.

Let $S$ be a topological space, and $\Phi$ a family of functions defined on $S$. The family $\Phi$ is said to be equi-continuous at a point $a$ of $S$, if for each positive $\varepsilon$, there is a neighbourhood $U$ of $a$ such that $|f(x)-f(a)|<\varepsilon$ for every $f \in \Phi$ and every $x$ of $U$. A family $\Phi$ of functions is equi-continuous on $S$, if $\Phi$ is equi-continuous at every point of $S$. Then we shall show the following proposition which was already obtained by I. Glicksberg [2]. ${ }^{1)}$

Proposition. A completely regular space $S$ is pseudo-compact, if and only if every pointwise bounded, equi-continuous family $\Phi$ contains a subsequence which converges uniformly on $S$.

Proof. Suppose that $S$ is not pseudo-compact, then there is an unbounded non-negative function $f(x)$ on $S$. Let $f_{n}(x)$ be the minimum function of $f(x)$ and $n$, i.e. $f_{n}(x)=\operatorname{Min}(f(x), n)$. Then $\left\{f_{n}(x)\right\}$ is pointwise bounded and we shall show that $\left\{f_{n}(x)\right\}$ is equi-continuous on $S$. For a point $a$ of $S$ and $\varepsilon>0$, there is a neighbourhood $U$ of $a$ such that $|f(x)-f(a)|<\varepsilon$ for each $x$ of $U$. For a positive integer $N$ such that $N \geq f(a)+\varepsilon$, we have $f_{n}(a)=f_{n}(x)(n \geq N)$ for $x$ of $U$. Taking a neighbourhood $V$ containing $a$ and contained in $U$ such that $\mid f_{i}(x)$ $f_{i}(a) \mid<\varepsilon$ for $x$ of $V$ and $i=1,2, \cdots, N-1$, then we have $\left|f_{n}(x)-f_{n}(a)\right|<\varepsilon$ for $x$ of $V$ and for every $f_{n}$.

Therefore $\left\{f_{n}\right\}$ is equi-continuous at the point $a$. This shows that $\left\{f_{n}\right\}$ is equi-continuous on $S$. On the other hand, we can not select a subsequence which converges uniformly on $S$, which contradicts. Hence $S$ is pseudo-compact.

To prove the converse, we shall show the following

Lemma. If a completely regular space $S_{1}$ is a continuous image

1) Recently, I found the paper by I. Glicksberg which is very available for the study of pseudo-compact spaces. His hypothesis for $\Phi$ is a bounded equi-continuous family. 
of a pseudo-compact completely regular space $S_{1}$, then $S_{1}$ is pseudocompact.

Proof. Since $S_{1}$ is completely regular, it is sufficient to prove that every countable open covering $\left\{O_{n}\right\}$ has the $A U$-property (see, S. Mardešić et P. Papić [5]). Let $f$ be the continuous mapping from $S$ onto $S$, then $f^{-1}\left(O_{n}\right)$ are open in $S$ and $\left\{f^{-1}\left(O_{n}\right)\right\}$ is a countable open covering of $S$. Hence we can find a finite system $\left\{f^{-1}\left(O_{n_{i}}\right)\right\}$ $(i=1,2, \cdots, k)$ such that $\bigcup_{i=1}^{k} \overline{f^{-1}\left(O_{n_{i}}\right)}=S$. Then continuity of $f$ implies $\overline{f^{-1}\left(O_{n_{i}}\right)} \subset f^{-1}\left(\bar{O}_{n_{i}}\right)$, and we have $S_{1}=f(S)=f\left(\bigcup_{i=1}^{k} \overline{f^{-1}\left(O_{n_{i}}\right)}\right) \subset \bigcup_{i=1}^{k} f\left(f^{-1}\left(\bar{O}_{n_{i}}\right)\right)$ $=\bigcup_{i=1}^{k} \bar{O}_{n_{i}}$. This shows that $S_{1}$ is pseudo-compact.

To prove the converse, we shall use the technique of I. Glicksberg, Therefore we shall review briefly the proof for the completeness. We shall use notations in the proof in the sequel. Let $\Psi=\left\{f_{n}(x)\right\}$ be a sequence of functions in $\Phi$, and we define $\rho(x, y)$ as

$$
\rho(x, y)=\sup _{n}\left|f_{n}(x)-f_{n}(y)\right| \text {. }
$$

Since $\left\{f_{n}(x)\right\}$ is pointwise bounded, $\rho(x, y)$ is finite for each pair $x, y$ of $S$. Therefore $\rho(x, y)$ defines a pseudo-metric on $S$. By the pseudometric $\rho$, we shall decompose $S$ into equivalence classes. We write $x \sim y$ whenever $\rho(x, y)=0$, i.e. $f_{n}(x)=f_{n}(y)$, then the relation " $\sim$ " is an equivalence relation. By $\mathbb{S}$, we denote the set of all equivalence classes. For $x$ of $S$, we write $\mathfrak{x}=F(x)$ for equivalence class which contains $x$. Then $\subseteq$ is a metric space under the metric $\rho(x, y)=\rho(x, y)$, where $\mathfrak{x}=F(x), \mathfrak{y}=F(y)$, and $F$ is continuous mapping from $S$ to $\mathfrak{S}$, since $\Psi$ is equi-continuous family. By Lemma, $\mathbb{S}$ is pseudo-compact, therefore $\mathfrak{S}$ is compact metric space. For each $f_{n}$ of $\Psi$, we define $F_{n}$ on $\subseteq$ by $F_{n}(\mathfrak{x})=f_{n}(x)$, where $\mathfrak{x}=F(x)$. For each $\mathfrak{x}$ of $\mathfrak{S}$. $\left\{F_{n}(\mathfrak{x})\right\}$ is bounded, and equi-continuous on $\subseteq$. By a diagonal method, we can select a subsequence $\left\{F_{n_{i}}(x)\right\}$ of $\left\{F_{n}(x)\right\}$ which converges uniformly to a continuous function $G(x)$ on $\subseteq$. Then $g(x)=G(F(x)$ ) is uniform limit of $F_{n_{i}}(x)$ on $S$.

Next, we shall consider a statement in Theorem 5.7 of R. G. Bartle $[1$, p. 49]. By using Lemma and notations above, we shall prove the following

Theorem 3. A completely regular space is pseudo-compact, if and only if every equi-continuous sequence which converges pointwisely to $a$ (continuous) function is uniformly convergent to it.

Proof. To prove the "if" part, ${ }^{2)}$ suppose that a completely regular space $S$ is not pseudo-compact, then there is a sequence $\left\{O_{n}\right\}$ of pairwise disjoint open sets such that $\left\{O_{n}\right\}$ has no cluster point. Let $a_{n}$ be in a point of $O_{n}$, then there is a continuous function $f_{n}(x)$ satisfy-

2) We can prove this part by the technique in Proposition. 
ing $0 \leq f_{n}(x) \leq 1, f_{n}\left(a_{n}\right)=1$ and $f_{n}(x)=0$ for $x \in S-O_{n}$. For a given point $x$, we can find a neighbourhood $U$ of $x$ such that $U$ meets only a finite system of $O_{n}$. This leads that $\left\{f_{n}\right\}$ is equi-continuous at $x$. On the other hand, $f_{n}(x)$ converges to 0 at every point and is not uniformly convergent to 0 . To prove the converse, let $\left\{f_{n}\right\}$ be an equi-continuous sequence, and let $f_{0}(x)$ be the limit function of the sequence $\left\{f_{n}(x)\right\}$. Suppose that $f_{n}(x)$ does not converge uniformly to $f(x)$ on $S$. Then there are a subsequence $\left\{f_{n_{i}}\right\}$ and a sequence $\left\{x_{i}\right\}$ of $S$ such that

$$
\left|f_{n_{i}}\left(x_{i}\right)-f_{0}\left(x_{i}\right)\right| \geq \varepsilon \quad(i=1,2, \cdots)
$$

for some fixed positive number $\varepsilon$. Since $\subseteq$ constructed above is compact metric space, $\mathfrak{x}_{i}=F\left(x_{i}\right)$ contains a convergent subsequence $\left\{\mathfrak{x}_{i_{k}}\right\}$ of $\left\{\mathfrak{x}_{i}\right\}$. We can suppose that $\mathfrak{x}_{i}$ converges to some point $\mathfrak{x}_{0}$ of $\subseteq$. Then $f_{n_{i}}\left(x_{i}\right) \rightarrow f_{n_{i}}\left(x_{0}\right)$, where $\mathfrak{x}_{0}=F\left(x_{0}\right) . \quad F_{n}(x)=f_{n}(F(x))$ is equi-continuous on $\subseteq$, then there is an integer $N$ such that $\left|F_{n_{i}}\left(\mathfrak{x}_{k}\right)-F_{n_{i}}\left(\mathfrak{x}_{0}\right)\right|<\varepsilon / 3$, i.e. $\left|f_{n_{i}}\left(x_{k}\right)-f_{n_{i}}\left(x_{0}\right)\right|<\varepsilon / 3$ for $i=1,2, \cdots$, and $k \geq N$ and $\left|F_{0}\left(x_{i}\right)-F_{0}\left(\mathfrak{x}_{0}\right)\right|$ $<\varepsilon / 3$, i.e. $\left|f_{0}\left(x_{i}\right)-f_{0}\left(x_{0}\right)\right|<\varepsilon / 3$ since $f_{0}$ is continuous. For sufficiently large $i, k$, we have

$$
\begin{aligned}
& \left|f_{n_{i}}\left(x_{k}\right)-f_{0}\left(x_{k}\right)\right| \leq\left|f_{n_{i}}\left(x_{k}\right)-f_{n_{i}}\left(x_{0}\right)\right| \\
& \quad+\left|f_{n_{i}}\left(x_{0}\right)-f_{0}\left(x_{0}\right)\right|+\left|f_{0}\left(x_{0}\right)-f_{0}\left(x_{k}\right)\right|<\varepsilon,
\end{aligned}
$$

by $f_{n_{i}}\left(x_{0}\right) \rightarrow f_{0}\left(x_{0}\right)$. This contradicts $(1)$.

\section{References}

[1] R. G. Bartle: On compactness in functional analysis, Trans. Amer. Math. Soc., 79, 35-37 (1955).

[2] I. Glicksberg: The representation of functionals by integrals, Duke Math. Jour., 19, 253-261 (1952).

[3] K. Iséki: A theorem on continuous convergence, Proc. Japan Acad., 33, 355356 (1957).

[4] C. Kuratowski: Topologie, I, 3rd edition, Warszawa (1952).

[5] S. Mardešić et P. Papić: Sur les espaces dont toute transformation réelle continue et bornée, Glasnik Mat.-Fiz. i. Astr., 10, 225-232 (1955). 